\title{
CORRECTION
}

\section{Correction to: Thoracic and cardiovascular surgery in Japan during 1998}

\author{
Committee of Science ${ }^{1} \cdot$ Keishu Yasuda ${ }^{2} \cdot$ Hiroyoshi Ayabe $^{3} \cdot$ Hiroko Ide $^{4} \cdot$ Yuzo Uchida $^{5}$
}

Published online: 15 June 2018

(c) The Japanese Association for Thoracic Surgery 2018

\section{Correction to: \\ General Thoracic and Cardiovascular Surgery 2000 https://doi.org/10.1007/BF03218167}

The original publication of the article cited above included incorrect values in the following parts: in Table 1 of section (B) Respiratory surgery and in Table 1 of section (C) Esophageal Surgery.

The corrected versions are shown below.

\section{(B) Re spiratory Surgery}

Table 1 Total entry cases of General Thoracic Surgery during 1998

\begin{tabular}{lrr}
\hline & Cases & \multicolumn{1}{c}{$\%$} \\
\hline Benign pulmonary tumor & 938 & 2.6 \\
Primary lung cancer & 15,468 & 42.4 \\
Other primary malignant pulmonary tumor & 173 & 0.5 \\
Metastatic pulmonary tumor & 2,620 & 7.2 \\
Tracheal tumor & 52 & 0.1 \\
Mesothelioma & 207 & 0.6 \\
Chest wall tumor & 525 & 1.4 \\
Mediastinal tumor & 2,494 & 6.8 \\
Thymectomy for MG without thymoma & 351 & 1.0 \\
Inflammatory pulmonary disease & 1,912 & 5.2 \\
Empyema & 914 & 2.5 \\
Bullous disease excluding pneumothorax & 1,243 & 3.4 \\
Pneumothorax & 8,292 & 22.7 \\
Chest wall deformity & 257 & 0.7 \\
Diapharagmatic hernia including traumatic & 127 & 0.3 \\
Chest trauma excluding diaphragmatic hernia & 252 & 0.7 \\
Others & 631 & 1.7 \\
Total & 36,456 & 100.0 \\
\hline
\end{tabular}

The original article can be found online at https://doi.org/10.1007/ BF03218167.

Committee of Science

survey-adm@umin.net

1 Tokyo, Japan

2 Department of Cardiovascular Surgery, Hokkaido University School of Medicine, Sapporo, Japan

3 First Department of Surgery, Nagasaki University School of Medicine, Nagasaki, Japan

4 Department of Surgery, Institute of Gastroenterolgy, Tokyo Women's Medical University, Tokyo, Japan

5 Department of Surgery II, Oita Medical University, Oita, Japan 


\section{(C) Esophageal Surgery}

Table 1 Benign esophageal diseases

\begin{tabular}{|c|c|c|c|c|c|}
\hline & \multicolumn{4}{|c|}{ Operation $(+)$} & \multirow[t]{3}{*}{ Operation $(-)$} \\
\hline & \multirow[t]{2}{*}{ Total cases } & \multirow[t]{2}{*}{ Endosc. surg } & \multicolumn{2}{|l|}{30 day death } & \\
\hline & & & Total cases & Endosc. surg & \\
\hline 1. Achalasia & 146 & 46 & 0 & 0 & 39 \\
\hline 2. Benign tumor & 173 & 65 & 0 & 0 & 80 \\
\hline (1) Leiomyoma & 116 & 42 & 0 & 0 & 59 \\
\hline (2) Cyst & 8 & 3 & 0 & 0 & 1 \\
\hline (3) Others & 49 & 20 & 0 & 0 & 20 \\
\hline 3. Diverticulum & 18 & 3 & 0 & 0 & 55 \\
\hline 4. Haital hernia & 270 & 80 & 1 & 1 & 458 \\
\hline 5. Spontaneous rupture of the esophagus & 41 & & 1 & & 8 \\
\hline 6. Esophageal perforation & 30 & & 3 & & 7 \\
\hline 7. Esophago-tracheal fistula & 24 & & 2 & & 21 \\
\hline 8. Congenital esophageal atresia & 80 & & 3 & & 5 \\
\hline 9. Congenital esophageal stenosis & 12 & & 0 & & 19 \\
\hline 10. Corrosive stricture of the esophagus & 10 & & 0 & & 7 \\
\hline 11. Esophagitis, esophageal ulcer & 47 & & 0 & & 531 \\
\hline 12. Esophageal varices & 269 & & 2 & & 1252 \\
\hline (1) Laparotomy & 198 & & 0 & & \\
\hline (2) Sclerotherapy & & & & & 1033 \\
\hline 13. Others & 37 & & 0 & 0 & 34 \\
\hline Total & 1157 & (194) & 13 & (1) & 2516 \\
\hline
\end{tabular}

